\title{
SMART CITIES Y ACCESIBILIDAD: LA NECESIDAD DE SUPERACIÓN DE NUEVAS FORMAS DE DISCRIMINACIÓN
}

\author{
Prof. Dr. Magdalena Suarez Ojeda \\ Profesora de Derecho Administrativo \\ Universidad Complutense de Madrid
}

Artículo Recibido: 06/04/2016

Artículo Aceptado: 01/06/2016

\section{Introducción}

El concepto de Smart City ha cobrado fuerza en los últimos años. Es inevitable que la revolución tecnológica que irrumpió en la vida de la ciudadanía en las últimas décadas de siglo pasado se viera traducido al espacio urbano en algún momento.

Así ha sido, el nuevo siglo comienza a integrar las nuevas tecnologías en el control de la ciudad que conlleva profundos cambios estructurales en lo que a la relación entre particulares y poderes políticos públicos se refiere. La posibilidad de interactuar con los servicios y de ser agentes activos del correcto funcionamiento de los poderes públicos ha abierto las puertas a un nuevo escenario de diálogo público-privado.

Pero las ciudades no había resuelto, ni mucho menos todos sus problemas previamente, la alta concentración de la población en las ciudades, el despoblamiento del campo, las infraviviendas, la polución, el tráfico y las emisiones de $\mathrm{CO} 2$, etc.

Entre estos problemas es digno de destacar las dificultades que tienen las personas con discapacidad para integrase en determinados espacios urbanos. Este problema puede verse amplificado por la introducción de nuevas tecnologías que expulsen directamente a una parte de la población de la marcha del mundo y del acceso a productos, bienes y servicios.

\section{1.- Dificultades de acceso de las personas con discapacidad}

Las personas con discapacidad se encuentran con diferentes tipos de barreras en la vida cotidiana: arquitectónicas (los impedimentos que se presentan en el interior de los edificios); urbanísticas (los impedimentos que presentan la estructura y el mobiliario urbano, lugares históricos y espacios no edificados de dominio público y privado); en el

WPS RI-SHUR, nํ3, 2016, vol.1, ISSN: 2387-1768 


\title{
WPSReview International on Sustainable
}

\author{
Housing and Urban Renewal (RI-SHUR)
}

transporte (los impedimentos que presentan las unidades de transporte particulares o colectivos, terrestres, marítimos, fluviales o aéreos), y en las telecomunicaciones (los impedimentos o dificultades que se presentan en la comprensión y captación de los mensajes, vocales y no vocales, y en el uso de los medios técnicos disponibles para las personas con diferente clase y grado de discapacidad).

Cuando se habla de discapacidad se hace referencia un amplio número de situaciones; éstas pueden ser permanentes o transitorias (accidentes o embarazo). Y afectar a condiciones psíquicas, físicas o cognitivas. De tal modo que dentro del grupo de personas con discapacidad hay tres grandes colectivos (Suárez, 2008):

1 Discapacidades permanentes derivadas de deficiencias físicas, sensoriales y mentales (personas discapacitadas permanentes de edad inferior a los 64 años)

2 Factores cronológicos (edad avanzada).

-Personas mayores discapacitadas.

-Personas mayores no discapacitadas.

Desde el punto de vista legal, hay una serie de instrumentos internacionales de protección de los derechos de este segmento de la población, entre los que destacan la Convención Internacional sobre los derechos de las personas con discapacidad, aprobada el 13 de diciembre de 2006 por la Asamblea General de las Naciones Unidas (ONU), Todo ello se ve avalado y multiplicado por la producción legal de la Unión Europea, ya que en la Carta de Derecho Fundamentales se incluye, sin ambages, los principios de no discriminación y la necesidad de acciones positivas en la integración de personas con discapacidad. Más allá de toda consideración, es indiscutible que la integración se ha convertido en un valor tanto social como jurídico, que es a la postre, el motor del cambio social, véase como el Preámbulo de la Carta dice lo siguiente: "la Unión está fundada sobre valores indivisibles y universales de la dignidad humana, la libertad, la igualdad y la solidaridad, y se basa en los principios de democracia y del Estado de Derecho [...] la Unión contribuye a la preservación y el fomento de los valores comunes. Así mismo el art. 13 del Tratado constitutivo de la Comunidad Europea habilita al Consejo para "adoptar acciones adecuadas para luchar contra la discriminación por motivos de sexo, de origen racial o étnico, religión o convicciones, discapacidad, edad u orientación sexual", en desarrollo de este precepto se han dictado numerosos documentos de soft law, además de la Declaración del 2003 como año Europeo de la Discapacidad. A lo se añade la aprobación de la directiva Directiva 2000/78/CE del Consejo, de 27 de noviembre de 2000, relativa al establecimiento de un marco general para la igualdad de trato en el empleo y la ocupación (DO L 303 de 2.12.2000) y la ratificación por parte de la Unión Europea del Convenio de Nueva York de 13 de diciembre de 2006 antes citado.

WPS RI-SHUR, n3, 2016, vol.1, ISSN: 2387-1768 


\title{
WPSReview International on Sustainable
}

\author{
Housing and Urban Renewal (RI-SHUR)
}

Es importante poner de manifiesto, que pese a lo que pueda pensarse hay un número muy significativo de personas que sufren algún tipo de discapacidad. Así, Un 10 por ciento de la población mundial, aproximadamente 650 millones de personas, vive con una discapacidad. Son la minoría más grande del planeta y, si se incluye a los miembros de sus familias, el número de personas directamente afectadas por la discapacidad asciende a alrededor de 2.000 millones, casi un tercio de la población mundial. (ONU) ${ }^{21}$."Se estima que más de mil millones de personas viven con algún tipo de discapacidad; o sea, alrededor del $15 \%$ de la población mundial (según las estimaciones del Resumen Informe mundial sobre la discapacidad la población mundial en 2010).

Esta cifra es superior a las estimaciones previas de la Organización Mundial de la Salud, correspondientes al año 1970, que eran de aproximadamente un 10\%. Según la última Encuesta Mundial de Salud, cerca de 785 millones de personas (15,6\%) de 15 años y más viven con una discapacidad, mientras que el proyecto sobre la Carga Mundial de Morbilidad estima una cifra próxima a los 975 millones $(19,4 \%)$. Señala también que del total estimado de personas con discapacidad, 110 millones $(2,2 \%)$ tienen dificultades muy significativas de funcionamiento, mientras que la Carga Mundial de Morbilidad cifra en 190 millones (3,8\%) las personas con una "discapacidad grave" (el equivalente a la discapacidad asociada a afecciones tales como la tetraplejía, depresión grave o ceguera). Solo la Carga Mundial de Morbilidad mide las discapacidades infantiles (0-14 años), con una estimación de 95 millones de niños $(5,1 \%), 13$ millones de los cuales $(0,7 \%)$ tienen "discapacidad grave" (Informe OMS personas con discapacidad, 2011).

En todo caso, hay un factor ineludible respecto de la población y es el envejecimiento. En este sentido la ONU se manifiesta así: "El mundo se encuentra en medio de un proceso irreversible y único de transición demográfica que resultará en poblaciones más envejecidas en todas partes. A medida que los índices de fertilidad disminuyen, se espera que la proporción de personas de 60 años o más se doble entre 2007 y 2050, y la cifra real será superior al triple, alcanzando los 2.000 millones en 2050. En la mayoría de los países, el número de personas de más de 80 años podría cuadruplicarse hasta alcanzar casi 400 millones para entonces."

\section{2.-Delimitación del entorno urbano respecto a la discapacidad}

La lucha contra la discriminación en el entorno urbano se plantea en tres grandes áreas: la urbanización, la accesibilidad en la edificación y plena integración en los transportes. Únicamente nos estamos refiriendo a la primera de ellas.

No se puede desconocer el esfuerzo de muchas ciudades por introducir desde hace años el concepto de un "diseño para todos" dentro de una perspectiva politológica

\footnotetext{
${ }^{21} \mathrm{http} / / /$ www.un.org/es/globalissues/disabilities/
}

WPS RI-SHUR, nํㅜ, 2016, vol.1, ISSN: 2387-1768 


\section{WPSReview International on Sustainable \\ Housing and Urban Renewal (RI-SHUR)}

inclusiva, en virtud de la cual todo sujeto debe dar de sí mismo todo lo que pueda e integrarse en la sociedad de forma activa, no solo por su propio bienestar -cuestión que es prioritaria- sino por el propio bien de la sociedad que aprende a vivir en sociedad con pluralidad de situaciones y se ejercita en el principio de "solidaridad entre las generaciones".

En el ámbito urbanístico los Ayuntamientos suelen tener un papel destacado en esta materia debiendo optimizar la calidad de los servicios mediante la innovación y la excelencia (Ayuntamiento de Madrid, 2012). La adaptación de la ciudad a los principios de accesibilidad universal comporta la necesidad de evaluar e integrar diferentes aspectos:

-En la prevención, promoción y mejora de la salud, se potencian e implantan programas de prevención de las principales discapacidades conocidas y de atención sanitaria especializada a grupos con necesidades especiales.

-En la educación, se realiza un esfuerzo por optimizar el acceso al conocimiento y la formación de las personas con discapacidad, asegurando que puedan acceder y permanecer en los distintos niveles del sistema educativo en igualdad de oportunidades, prestando una atención especial a la transición de las personas con discapacidad a la etapa adulta.

- En el empleo, se potencian medidas de garantía e inclusión en el empleo público, autónomo y en la empresa privada.

- En la participación social de las personas con discapacidad se avanza para conseguir la plena integración de todas las personas con discapacidad y sus familias. En su caso, resulta obligado diseñar un área específica que engloba las principales acciones para la atención temprana, prestando una atención especial a la coordinación de los servicios destinados a la atención de los niños entre 0 y 6 años así como el apoyo a sus familias.

Para que todo esto sea un hecho, en el ámbito que nos ocupa es necesario evaluar de modo correcto los obstáculos que actualmente tienen las ciudades. Estos pueden clasificarse en los siguientes parámetros (Moroni, 2004):

Barreras urbanísticas permanentes:

-Aceras estrechas, muy frecuentes en cascos antiguos, situación que se agrava cuando existe cualquier otro elemento transitorio o temporal reduce aún más su ancho.

- Calles con demasiada pendiente.

- Pavimentos rotos o calles sin pavimentar.

- Encintados altos.

- Árboles con ramas bajas.

- Toldos de locales comerciales.

WPS RI-SHUR, nํㅜ, 2016, vol.1, ISSN: 2387-1768 


\section{WPSReview International on Sustainable}

\section{Housing and Urban Renewal (RI-SHUR)}

- Postes con tensores sin protección.

- Refugios para paradas de autobuses a los cuales se le adosan publicidad.

- Portones o puertas, medidores de luz o acondicionadores de aire que abren hacia fuera

- Estaciones de servicio (gasolineras) con dificultoso paso para peatones en general.

- Sillas y mesas de restaurantes, casetas de venta de periódicos, etc.

Barreras urbanísticas temporales:

- Obras, públicas o privadas sin la señalización correspondiente.

- Obstáculos a cierta altura que sobresalen zanjas y pozos sin cerrar.

- Andamios, especialmente los metálicos que se instalan para reparar fachadas y ocupan prácticamente toda la acera y, a veces, dejan un pequeño espacio por debajo.

- Tapas de bocas de tormenta.

- Bolsas de basura, escombros, motos y coches estacionados sobre la acera, carteles anunciadores entre otros.

\section{3.-Smart Cities y accesibilidad}

Los problemas que acabamos de relatar no están completamente solventados en los entornos urbanos. Pero en la actualidad nos encontramos con nuevo elemento de análisis, la introducción de las nuevas tecnologías en la gestión de las ciudades. Indudablemente, ello supone una situación esperanzadora por el impulso que puede suponer en el progreso de la disciplina. Pero junto con las luces llegan las sombras. No se puede soslayar que muchas personas no están familiarizadas con el entorno digital (en lo que cobra importancia la brecha por edad) o carecen de medios económicos suficientes para adquirir los mecanismos adecuados para incorporarse a los progresos técnicos.

Por otro lado, se observa una creciente competitividad entre las ciudades, el "efecto llamada" del concepto smart cities hace imperiosa la introducción de alguna medida dinamizadora y claramente visible (cuando no aparatosa) de incorporación al "nuevo escenario digital". Con todo, no se puede olvidar que la gestión de las grandes ciudades exigen un ingente esfuerzo económico que en la mayor parte de los casos es imposible abordar de modo global. Ante ello se han propuesto soluciones intermedias como dotar de tecnología punta determinados barrios (principalmente de nueva urbanización o el casco histórico) dejando para un segundo momento su aplicación al conjunto de la ciudad, lo que supone de modo inmediato un nuevo elemento de diferencia/segregación social.

En este sentido hay una serie de medidas que ya son realidad en muchos entornos urbanos, que intentan dar solución a los problemas de integración. Entre ellas, paso a señalar algunas que han sido implantas con éxito, a saber:

WPS RI-SHUR, nํㅜ, 2016, vol.1, ISSN: 2387-1768 


\section{WPSReview International on Sustainable \\ Housing and Urban Renewal (RI-SHUR)}

-Elaboración de un correcto censo de poblaciones que permita determinar las limitaciones cognitiva, físicas y sensoriales haciendo un estudio de diseño tecnológico preciso para la superación de barreras en función del tipo de discapacidad.

-Educación en nuevas tecnologías pero teniendo en cuenta que la implantación de las smart cities habrá siempre un conjunto de personas que por edad o condición económica y social jamás serán usuarios de las TICS.

-Asistencia social 24 horas que permita con una simple llamada de teléfono o pulsación de un sencillo mecanismo la activación de los servicios de urgencia. ${ }^{22}$

-Adaptación de las ciudades para la presencia de perros-guía utilizados por personas con déficit visual.

-En el caso de que las ciudades opten por la introducción de la bicicleta como método alternativo a los vehículos de motor, sería aconsejable que un número de ellos

-Correcta señalización de las calles, que permita a cualquier persona identificar el lugar donde su encuentra. En el caso de que las personas sufran desorientación es aconsejable instalación de sistemas de fácil pulsión que active servicios sociales y/o de seguridad.

-Propiciar el estilo de vida activo creando espacios abiertos y seguros para la práctica de deporte (running) o instalación de elementos gimnásticos para adultos en parques.

- Generar un sistema de transportes de recorridos accesibles, como sistemas guiados en las redes de metro $y$ tren

-Servicios de avisos al conductor de medios de transportes a través de tarjetas chips que avisen del lugar donde debe bajarse la persona invidente

-Establecimiento de semáforos por segundos que permitan determinar con exactitud el tiempo de espera y valorar si capacidad suficiente para abordar el cruce una vía. Ello incluye la necesidad de establecimiento de medidas acústicas.

-Adaptación de las marquesinas de los autobuses, creación de plataformas de espera adecuadas para transporte urbano en carreras, así como medidas de accesibilidad especiales en travesías

-Utilización de la robótica en sentido omnicomprensivo al servicio de la accesibilidad de la ciudad

-Buscadores que detectan dificultades para la movilidad de personas con discapacidad

WPS RI-SHUR, nํㅜ, 2016, vol.1, ISSN: 2387-1768 


\section{WPSReview International on Sustainable \\ Housing and Urban Renewal (RI-SHUR)}

\section{4.-Conclusion}

La principal conclusión es que el nuevo concepto de smart cities no debe convertirse en una nueva forma de discriminación para ello debe estar alerta e instaurar procesos y servicios inclusivos, teniendo en cuenta el significativo número de personas que sufren discapacidad y en envejecimiento de la población.

Para ello cada ciudad debería introducir unas medidas de corte jurídico-politológico adecuado que permitan saber con precisión cuanta personas sufren discapacidad y de qué tipo. Cuidando que las directrices adoptadas sean también aplicables a todas las políticas utilizando mecanismos como el mainstreaming y la interseccionalidad.

Es conveniente también insistir en la formación de las poblaciones tanto en valores inclusivos y establecer un sistema sencillo para el manejo de las nuevas tecnológicas.

Finalmente hay que resaltar, que en cuestiones de corte social, no se resuelven únicamente con grandes inversiones económicas -cuestión que es decisiva por supuesto-, sino también de sensibilidad y toma de conciencia de la situación, pensando realmente que el diseño y los servicios deben estar dirigidos para todas las personas.

\section{BIBLIOGRAFIA}

-Moroni, C.P. "Barreras urbanísticas" en "Discapacidad visual hoy aportes sobre la visión diferenciada" http://www.juntadeandalucia.es/averroes/caidv/interedvisual/dvh_12/dvh_12_6.pdf

- Informe mundial sobre la discapacidad 2011. Organización Mundial de la Salud y Banco Mundial (http://www.who.int/disabilities/world_report/2011/summary_es.pdf)

-Suárez, M. (2009) "La accesibilidad en la edificación: reflexión general y nuevas perspectivas" Revista Aranzadi de Urbanismo y Edificación nº 18: 43-52.

-Thite, M. (2011) "Smart cities: implications of urban planning for human resource Human Resource" Development International Vol. 14, No. 5, November 2011: 623-631

-Thuzar, Moe (2011/2012) "Urbanization in south Asia: developing smart cities for the future? Regional Outlook 2011/2012 Southeas: 96-100.

-Australian Urban Research Infrastructure Network (AURIN) (20149 to release new data analysis tools for smart cities. CIO (13284045)) "Residential segregation among social and ethnic groups in Budapest during the post- communist transition", in $\mathrm{P}$ Marcuse, $\mathrm{R}$ van Kempen (eds) Of States and Cities: The Partitioning of Urban Space .Oxford University Press, Oxford: 170-182.

WPS RI-SHUR, n3, 2016, vol.1, ISSN: 2387-1768 